\title{
Dynamical localization of matter-wave solitons in managed barrier potentials
}

\author{
Fatkhulla Kh. Abdullaev ${ }^{1}$ and Josselin Garnier ${ }^{2}$ \\ ${ }^{1}$ Physical-Technical Institute of the Uzbekistan Academy of Sciences, 700084, Tashkent-84, G.Mavlyanov str., 2-b, Uzbekistan \\ and Instituto de Física Teorica, UNESP, Rua Pamplona, 145, São Paulo, Brazil \\ ${ }^{2}$ Laboratoire de Probabilités et Modèles Aléatoires \& Laboratoire Jacques-Louis Lions, Université Paris VII, 2 Place Jussieu, \\ 75251 Paris Cedex 5, France
}

(Received 29 September 2006; revised manuscript received 1 December 2006; published 8 March 2007)

The bright matter-wave soliton propagation through a barrier with a rapidly oscillating position is investigated. The averaged-over rapid oscillations Gross-Pitaevskii equation is derived, where the effective potential has the form of a finite well. Dynamical trapping and quantum tunneling of the soliton in the effective finite well are investigated. The analytical predictions for the effective soliton dynamics is confirmed by numerical simulations of the full Gross-Pitaevskii equation.

DOI: $10.1103 /$ PhysRevA.75.033603

PACS number(s): 03.75.Lm, 02.30.Jr, 05.45.Yv, 42.65.Tg

\section{INTRODUCTION}

The dynamical localization and tunneling of quantum particles in oscillating in time potentials is one of the problems of quantum mechanics that has attracted a lot of attention recently $[1,2]$. In particular, it is interesting to study the localization and transmission phenomena under action of timedependent barriers. Indeed, experiments with dynamical tunneling of cold atoms in time-dependent barriers of the multiplicative form $f(t) V(x)$ show the possibility for the tunneling period control $[3,4]$. The theory is developed for linear wave packets in Ref. [5]. Soliton propagation through such a barrier is investigated in Ref. [6]. Another interesting problem is to consider a barrier with a rapidly oscillating position, i.e., a potential of the form $V[x-f(t)]$. In the linear regime of propagation resonant effects are exhibited in the transmission process of matter wave packets through an oscillating barrier $[7,8]$. The split of repulsive condensate under a trap with an oscillating position has been investigated in Ref. [9]. The dynamical localization of optical wave in the array of periodically curved optical waveguides has been demonstrated recently in Refs. $[10,11]$. This system is described by a nonlinear Schrödinger- (NLS) type equation with a periodic potential with an oscillating position. It is therefore relevant to investigate the propagation of a nonlinear wave packet and, in particular, the dynamical localization of wave packets in barriers with time-dependent parameters. We consider this problem for a bright matter-wave soliton in an attractive Bose-Einstein condensate (BEC) in the presence of a barrier potential with an oscillating position. Such a barrier can be achieved by using a laser beam with a bluedetuned far-off-resonant frequency. This generates a repulsive potential and the laser sheet plays the role of a mirror for cold atoms. Such a laser sheet has been used to study the bouncing of a BEC cloud off a mirror [12]. By moving the position of the mirror we obtain an oscillating barrier.

In this paper we are mainly interested in the study of the dynamical trapping of soliton in BEC with a rapidly oscillating barrier potential. The averaged-over rapid oscillations Gross-Pitaevskii (GP) equation is derived in Sec. II, and we show that the effective potential has the form of a finite well. Applying a variational approach to this equation, we analyze the conditions for soliton trapping by the effective potential in Sec. III. The analytical predictions are checked by numerical simulations of the full GP equation in Sec. IV. We analyze carefully the case of broad solitons in Sec. V. In Sec. VI we make the important remark that the management technique based on rapid oscillations of a barrier potential is very flexible and allows the generation of asymmetric traps. As a consequence, this technique can be used to investigate soliton tunneling with sub-barrier energies as we now explain. In Refs. $[13,14]$ the topological soliton tunneling in an asymmetric well with a finite barrier was investigated. In this paper we analyze a similar problem for the NLS soliton case and show that the tunneling in our case has the form of continuous shedding of tunneling radiation by the soliton (due mainly to the fact that the NLS soliton is the dynamical soliton) and formation in the effective well of breather-type solutions. This phenomenon represents a quantum evaporation of the soliton from the dynamically generated potential.

\section{AVERAGED GROSS-PITAEVSKII EQUATION}

The BEC wave function $\psi$ in a quasi-one-dimensional geometry is described by the GP equation,

$$
i \hbar \psi_{T}+\frac{\hbar^{2}}{2 m} \psi_{X X}-V_{p}\left[X-f_{p}(T), T\right] \psi-g_{1 D}|\psi|^{2} \psi=0,
$$

where $V_{p}(X, T)$ is a barrier potential. Here $g_{1 D}=2 \hbar a_{s} \omega_{\perp}$ is the one-dimensional mean-field nonlinearity constant, $a_{s}$ is the atomic scattering length, $\omega_{\perp}$ is the transverse oscillator period, and $a_{\perp}=\sqrt{\hbar /\left(m \omega_{\perp}\right)}$ is the transverse oscillator length. Below we consider the case of BEC with attractive interactions, i.e., $a_{s}<0$. To avoid collapse in the attractive BEC, the condition $\left|a_{s}\right| N / a_{\perp}<0.67$ should be satisfied, with $N$ the number of atoms. The barrier potential has an oscillating position described by the zero-mean time-periodic function $f_{p}(T)$ with period $T_{p}$. We consider the case of a fastmoving potential in the sense that $\varepsilon:=\omega_{\perp} T_{p} \ll 1$. We also allow the barrier potential profile $V_{p}(X, T)$ to vary slowly in time, i.e., with a rate smaller than $\omega_{\perp}$. Introducing the standard dimensionless variables 


$$
x=\frac{\sqrt{2} X}{a_{\perp}}, \quad t=\omega_{\perp} T, \quad u=\sqrt{\left|a_{s}\right|} \psi, \quad V=\frac{V_{p}}{\hbar \omega_{\perp}},
$$

we obtain the GP equation with a fast-moving potential

$$
i u_{t}+u_{x x}-V\left[x-f\left(\frac{t}{\varepsilon}\right), t\right] u+2|u|^{2} u=0
$$

where

$$
f(t)=\frac{\sqrt{2}}{a_{\perp}} f_{p}\left(T_{p} t\right)
$$

is a periodic function with period 1 and mean 0 . The small parameter $\varepsilon$ describes the fast oscillation period in the dimensionless variables. We look for the solution in the form

$$
u(x, t)=u^{(0)}\left(x, t, \frac{t}{\varepsilon}\right)+\varepsilon u^{(1)}\left(x, t, \frac{t}{\varepsilon}\right)+\varepsilon^{2} u^{(2)}\left(x, t, \frac{t}{\varepsilon}\right)+\cdots,
$$

where $u^{(0)}, u^{(1)}, \ldots$ are periodic in the argument $\tau=t / \varepsilon$. We substitute this ansatz into Eq. (1) and collect the terms with the same powers of $\varepsilon$. We obtain the hierarchy of equations,

$$
\begin{gathered}
i u_{\tau}^{(0)}=0, \\
i u_{t}^{(0)}+u_{x x}^{(0)}-V[x-f(\tau), t] u^{(0)}+2\left|u^{(0)}\right|^{2} u^{(0)}=-i u_{\tau}^{(1)}, \\
i u_{t}^{(1)}+u_{x x}^{(1)}-V[x-f(\tau), t] u^{(1)}+4\left(u^{(0)}\right)^{2} \overline{u^{(1)}}+2\left|u^{(0)}\right|^{2} u^{(1)} \\
=-i u_{\tau}^{(2)} .
\end{gathered}
$$

The first equation (3) shows that the leading-order term $u^{(0)}$ does not depend on $\tau$. The second equation (3) gives the compatibility equation for the existence of the expansion (2),

$$
i u_{t}^{(0)}+u_{x x}^{(0)}-\langle V[x-f(\cdot), t]\rangle u^{(0)}+2\left|u^{(0)}\right|^{2} u^{(0)}=0,
$$

where $\langle\cdot\rangle$ stands for an average in $\tau$. Denoting

$$
V_{\mathrm{eff}}(x)=\int_{0}^{1} V[x-f(\tau), t] d \tau,
$$

we obtain the averaged GP equation

$$
i u_{t}+u_{x x}-V_{\text {eff }}(x, t) u+2|u|^{2} u=0 .
$$

Equation (5) allows us to compute the first-order correction, whose amplitude is of order $\varepsilon$.

If, for example, $f(\tau)=a \sin (2 \pi \tau)$, then a straightforward calculation shows that

$$
V_{\mathrm{eff}}(x, t)=K_{\mathrm{eff}} * V(x, t),
$$

where $*$ stands for the convolution product (in $x$ ) and $K_{\text {eff }}$ is given by

$$
K_{\mathrm{eff}}(x)=\frac{1}{\pi} \frac{1}{\sqrt{a^{2}-x^{2}}} \mathbf{1}_{(-a, a)}(x)
$$

If, moreover, $V(x, t)$ is a deltalike potential centered at a position $x_{0}(t)$ that moves slowly in time, then

$$
V_{\text {eff }}(x, t)=K_{\text {eff }}\left[x-x_{0}(t)\right]
$$

is a moving double-barrier potential. By this way, one can generate a moving trap potential to manage the soliton position.

\section{VARIATIONAL APPROACH FOR THE SOLITON MOTION}

In the absence of effective potential $V_{\text {eff }}=0$ the NLS equation (7) supports soliton solutions of the form

$$
u_{0}(x, t)=2 \nu \frac{e^{i 2 \mu\left[x-x_{s}(t)\right]+4 i\left(\mu^{2}+\nu^{2}\right) t}}{\cosh \left\{2 \nu\left[x-x_{s}(t)\right]\right\}},
$$

with $x_{s}(t)=x_{s}(0)+4 \mu t$. In the presence of a stationary potential $V_{\text {eff }}(x)$, the soliton dynamics can be studied by perturbation techniques. Applying the first-order perturbed inverse scattering transform (IST) theory [17], we obtain the system of equations for the soliton amplitude and velocity

$$
\frac{d \nu}{d t}=0, \quad \frac{d \mu}{d t}=-\frac{1}{4 \nu} W_{\nu}^{\prime}\left(x_{s}\right), \quad \frac{d x_{s}}{d t}=4 \mu,
$$

where the prime stands for a derivative with respect to $x$ and $W_{\nu}$ has the form

$$
W_{\nu}(x)=\nu \int_{-\infty}^{\infty} \frac{1}{\cosh ^{2}(z)} V_{\mathrm{eff}}\left(x-\frac{z}{2 \nu}\right) d z=K_{\nu} * V_{\mathrm{eff}}(x),
$$

$$
K_{\nu}(x)=\frac{2 \nu^{2}}{\cosh ^{2}(2 \nu x)}
$$

Therefore, we can write the effective equation describing the dynamics of the soliton center as a quasiparticle moving in the effective potential $W_{\nu_{0}}$,

$$
\nu_{0} \frac{d^{2} x_{s}}{d t^{2}}=-W_{\nu_{0}}^{\prime}\left(x_{s}\right)
$$

where $4 \nu_{0}$ is the mass (number of atoms) of the initial soliton. This system has the integral of motion

$$
\frac{\nu_{0}}{2}\left(\frac{d x_{s}}{d t}\right)^{2}+W_{\nu_{0}}\left(x_{s}\right)=8 \nu_{0} \mu_{0}^{2}
$$

where $4 \mu_{0}$ is the velocity of the initial soliton. Note that the first-order perturbed IST takes into account the nonlinear wave nature of the soliton, but neglects radiations phenomena by considering that the matter wave remains in the form of a soliton with time-dependent coefficients (velocity, center, and phase). This approach gives the same result as the adiabatic perturbation theory for solitons, which is a firstorder method as well. This adiabatic perturbation theory was originally introduced for optical solitons $[18,19]$ and it was recently applied to matter-wave solitons [20].

The quasiparticle effective potential $W_{\nu}$ is given by 


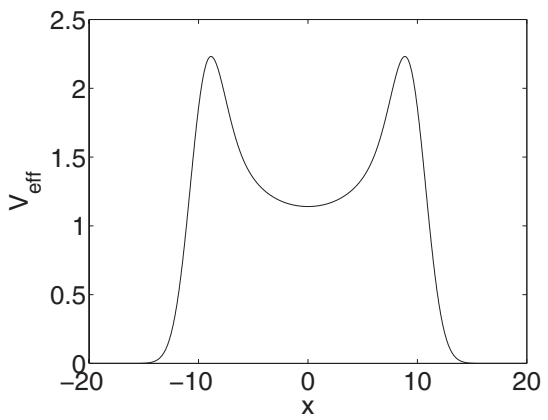

$$
W_{\nu}(x)=\left(K_{\nu} * K_{\mathrm{eff}}\right) * V(x) .
$$

In the case where $f(\tau)=a \sin (2 \pi \tau)$, the kernel $K_{\text {eff }}$ is given by Eq. (9) and the Fourier transform of the quasiparticle potential has the explicit form

$$
\hat{W}_{\nu}(k)=\frac{\pi k J_{0}(k a)}{2 \sinh \left(\frac{\pi k}{4 \nu}\right)} \hat{V}(k),
$$

where $\hat{V}$ is the Fourier transform of the reference potential $V$. The effective quasiparticle potential is plotted in Fig. 1 for a Gaussian barrier potential $V$. The potential $W_{\nu}$ has a local minimum at $x=0$ between two global maxima that are close to $x= \pm a$. The trap amplitude is

$$
\Delta W_{\nu}=\max _{x} W_{\nu}(x)-W_{\nu}(0) .
$$

Let us consider an input soliton at $x=0$ with parameters $\left(\nu_{0}, \mu_{0}\right)$. If the initial soliton velocity is large enough, then the soliton escapes the trap. There is a critical value $\mu_{c}$ for the initial soliton velocity parameter $\mu_{0}$ defined by

$$
\mu_{c}^{2}=\frac{\Delta W_{\nu_{0}}}{8 \nu_{0}}
$$

which determines the type of motion.

(1) If $\left|\mu_{0}\right|<\mu_{c}$ then the soliton is trapped. Its motion is oscillatory between the positions $\pm x_{f}$ defined by

$$
W_{\nu_{0}}\left(x_{f}\right)-W_{\nu_{0}}(0)=8 \nu_{0} \mu_{0}^{2} .
$$

(2) If $\left|\mu_{0}\right|>\mu_{c}$, then the soliton motion is unbounded. It escapes the trap and reaches the asymptotic velocity parameter $\mu_{a}$ given by

$$
\mu_{a}^{2}=\frac{W_{\nu_{0}}(0)}{8 \nu_{0}}+\mu_{0}^{2},
$$

which shows that the transmitted soliton velocity is larger than the initial soliton velocity.

As we shall see in the numerical simulations, if the initial soliton parameters are close to the critical case $\left|\mu_{0}\right| \sim \mu_{c}$, then radiation effects become non-negligible. The construction of an efficient trap requires one to generate a barrier potential $V$ that is high enough so that $\Delta W_{\nu_{0}}$ is significantly larger than $8 \nu_{0}^{2} \mu_{0}$.
FIG. 1. Effective potentials $V_{\text {eff }}(x)$ for the averaged equation (7) and $W_{\nu}(x)$ for the quasiparticle motion (12). Here $\nu=0.25, V(x)$ $=10 \exp \left(-x^{2} / 4\right)$, and $f(\tau)=10 \sin (2 \pi \tau)$.

\section{NUMERICAL SIMULATIONS FOR DYNAMICAL LOCALIZATION}

In this section we show the motion of a soliton with initial parameters $\nu_{0}=\mu_{0}=0.25$ (the soliton velocity is 1 ). The reference barrier potential is $V(x)=10 \exp \left(-x^{2} / 4\right)$. Note that in this case, $\Delta W_{\nu_{0}} \simeq 0.323$ and $\mu_{c} \simeq 0.4$. Therefore, the quasiparticle approach predicts trapping.

In Fig. 2 the modulated potential is $V[x-10 \sin (50 t)]$ (stationary trap). Here $\varepsilon=2 \pi / 50 \simeq 0.126$ and we check by full numerical simulations of the GP equation (1) that $\varepsilon$ is small enough to ensure the validity of the theoretical predictions based on the averaged GP equation (7). We plot the soliton profile and the soliton mass, which shows that radiative effects are very small. We also compare the soliton motion obtained by the numerical simulations with the one predicted by the quasiparticle approach, which shows very good agreement.

In Fig. 3 the modulated potential is $V[x-10 \sin (50 t)$ $-0.25 t$ ] (moving trap, with velocity 0.25 ). We plot the soliton profile and the soliton mass, which shows that radiative effects are very small.

In Fig. 4 the modulated potential is $V[x-10 \sin (50 t)$ $+0.25 t]$ (moving trap, with velocity -0.25 ). Radiation effects are small, but not completely negligible. It can be seen that some radiation is transmitted through the edges of the effective double-barrier trap. We then double the potential amplitude and consider $2 V[x-10 \sin (50 t)+0.25 t]$. The new simulation is shown in Fig. 5, where radiation effects are seen to be negligible.

In Fig. 6 we show the motion of a soliton with initial parameters $\nu_{0}=0.25$ and $\mu_{0}=0.5$ (the soliton velocity is 2 ). Since we have $\Delta W_{\nu_{0}} \simeq 0.323$ and $\mu_{c}=0.4$, the quasiparticle approach predicts unbounded motion. The results of the numerical simulations confirm this prediction. We can observe that half the initial soliton mass is transmitted through the effective barrier during the first interaction, and the transmitted soliton has a velocity that is larger than $4 \mu_{0}$.

Let us estimate the typical values of the parameters for an experimental configuration. We consider the case of a condensate with ${ }^{7} \mathrm{Li}$ atoms in the a cigar-shaped trap potential with $\omega_{\perp}=2 \pi \times 1 \mathrm{kHz}$. In this case, $\varepsilon=2 \pi / 50$ corresponds to 

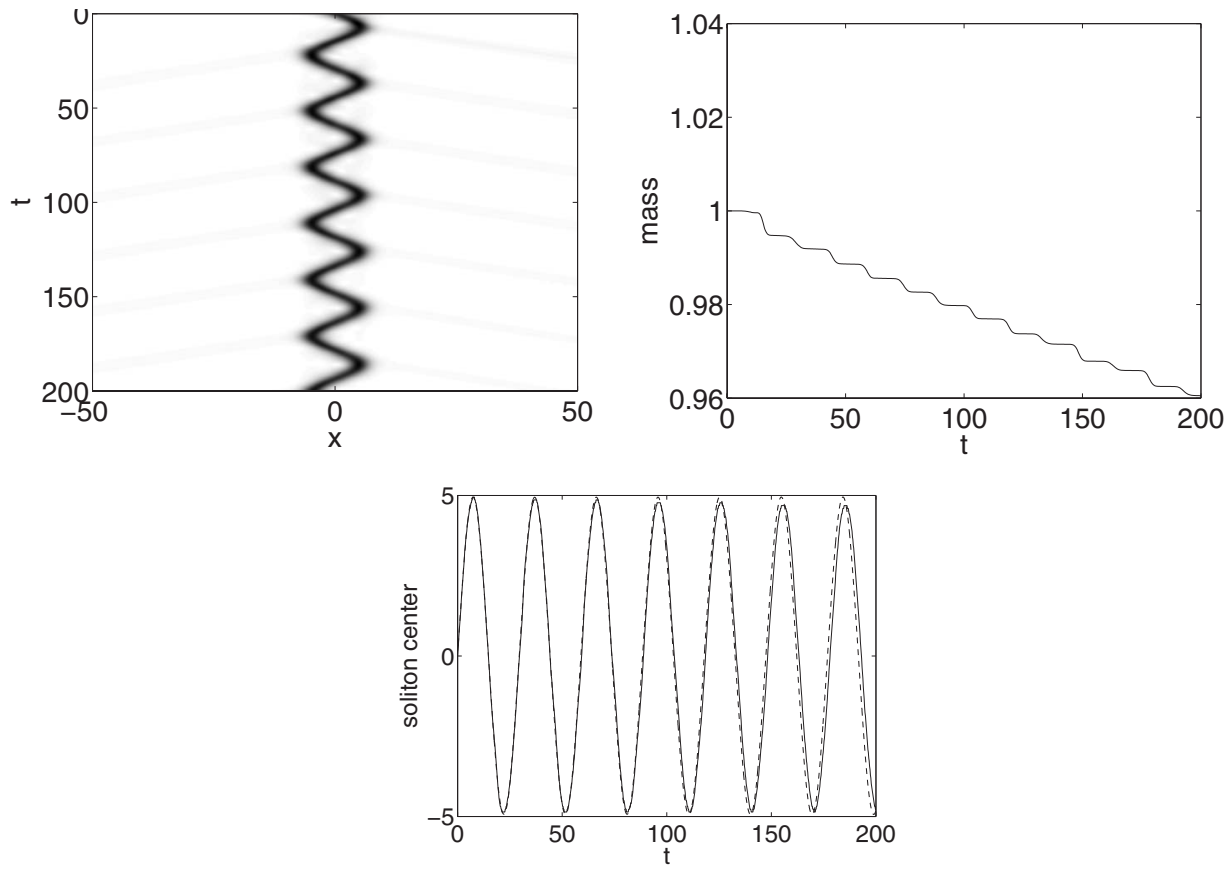

$1 / T_{p}=50 \mathrm{kHz}$. The barrier height is $V_{0}=10 \hbar \omega_{\perp}$. The number of atoms can be taken as $N \sim 10^{3}$ for the scattering length $a_{s}=-0.2 \mathrm{~nm}$. The transverse oscillator length is $a_{\perp}=1.2 \mu \mathrm{m}$, so the barrier width is $d \simeq 2 \mu \mathrm{m}$ and the oscillations amplitude of the barrier position is $L \simeq 10 \mu \mathrm{m}$. The velocity is normalized by the sound velocity, which is $c \simeq 4 \mathrm{~mm} / \mathrm{s}$, so the soliton velocity parameter $\mu_{0}=0.25$ corresponds to a velocity equal to $c$.
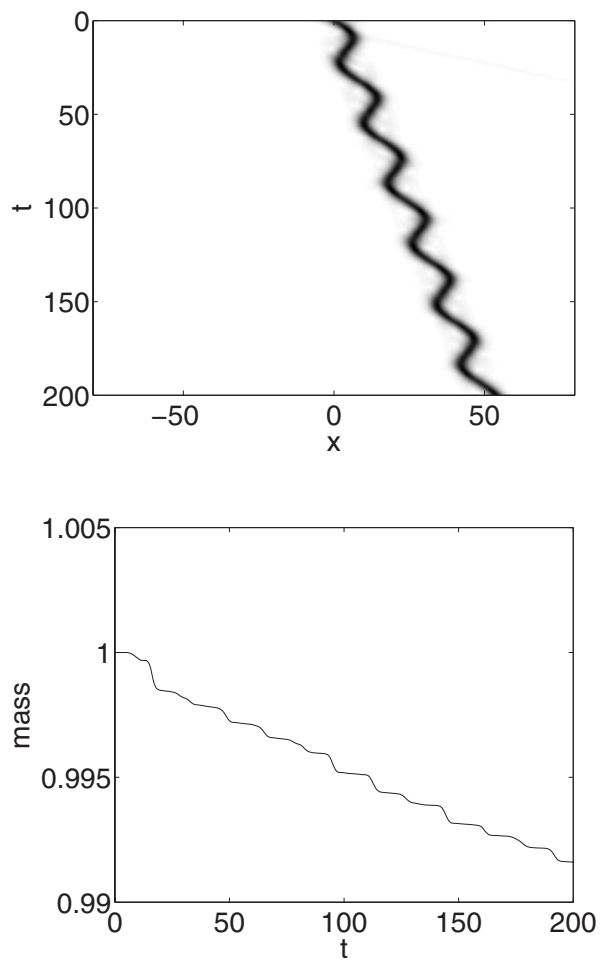

FIG. 3. Soliton profile in a narrow moving trap $V[x-10 \sin (50 t)-0.25 t]$ (top). Mass decay (bottom).
FIG. 2. Soliton profile in a narrow trap $V[x-10 \sin (50 t)]$ (top left). Mass decay (top right). Bottom: Soliton motion predicted by the quasiparticle approach (dashed) and observed in the numerical simulations (solid).
If the soliton is broad and its width becomes comparable to (or larger than) the width of the trap generated by the effective potential $V_{\text {eff }}$, in the sense that $2 \nu a \sim 1$, then the quasiparticle potential $W_{\nu}$ does not present any local minimum (see Fig. 7). Indeed, by Eq. (10) the quasiparticle potential $W_{\nu}$ is the convolution of the effective potential with
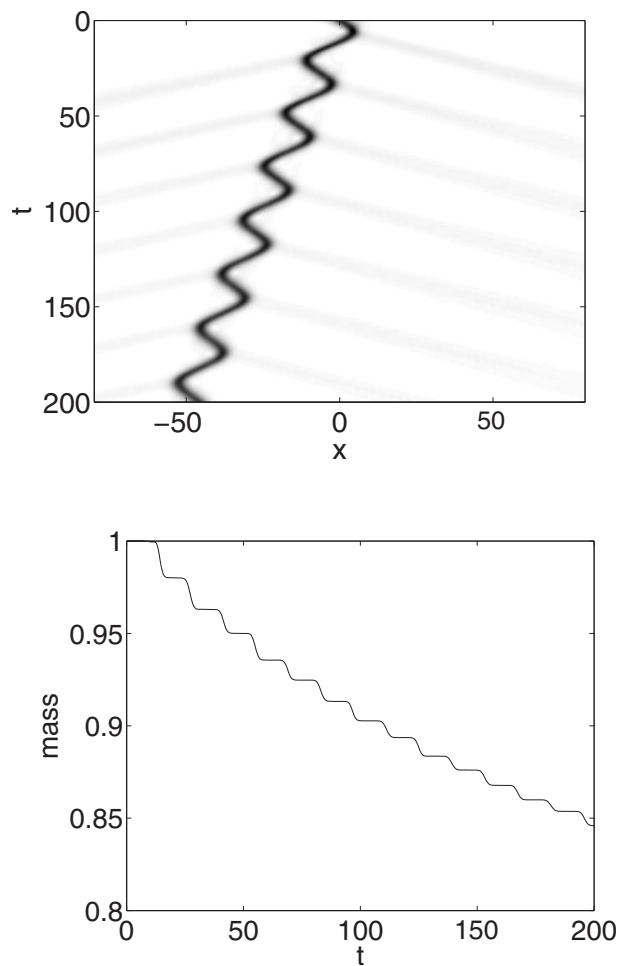

FIG. 4. Soliton profile in the moving trap $V[x-10 \sin (50 t)$ $+0.25 t]$ (top). Mass decay (bottom). 

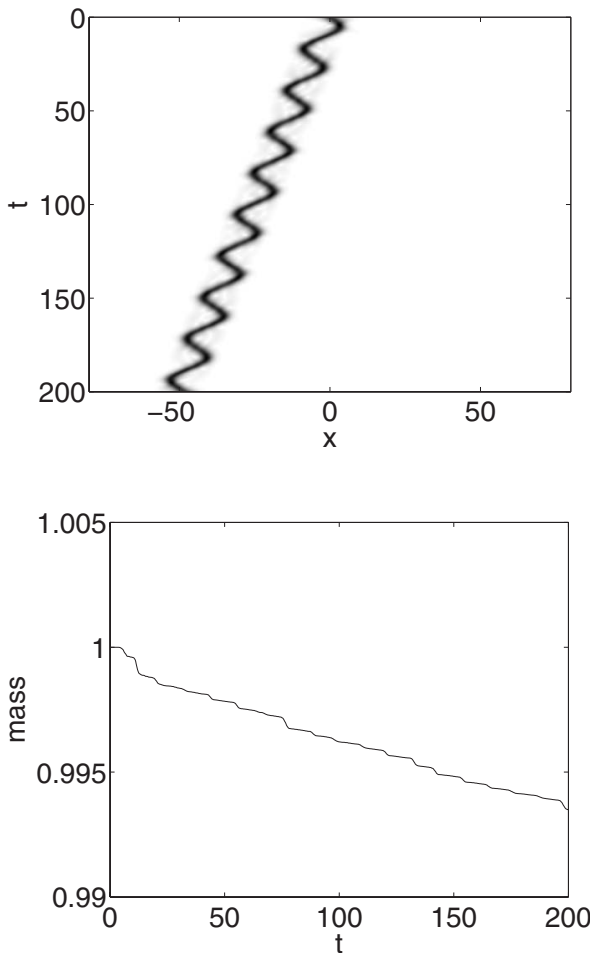

FIG. 5. Soliton profile in the moving trap $2 V[x-10 \sin (50 t)$ $+0.25 t]$ (top). Mass decay (bottom).

the convolution kernel $K_{\nu}$, which is a sech [2] function scaled by $\nu$. As a result, for large $\nu$, i.e., for narrow soliton, the quasiparticle potential $W_{\nu}(x)$ resembles the effective potential $V_{\text {eff }}(x)$. For small $\nu$, i.e., for broad soliton, the convolution with $K_{\nu}$ smooths the trap, the local minimum is washed out, and the quasiparticle potential has the form of a broad Gaussian barrier centered at 0 . In this case, a soliton centered at 0 with a nonzero velocity escapes the trap. A soliton centered at 0 with initial zero velocity $\mu_{0}=0$ has a different dynamics. The quasiparticle approach predicts an absence of motion. However, for long times, the soliton emits radiation and then becomes a Gaussian-type breather whose width periodically oscillates in the trap $V_{\text {eff }}$. After a long time of the order of a few thousands, these oscillations are damped and the solution converges to a stationary Gaussian-type wave function. The periodic oscillations and the asymptotic Gaussian wave function can be predicted analytically. The effective potential $V_{\text {eff }}$ can be fitted by the quadratic trap

$$
V_{\mathrm{eff}}(x)=V_{0}+V_{1} x^{2}
$$

where

$$
\begin{aligned}
V_{1}=\frac{1}{2} V_{\mathrm{eff}}^{\prime \prime}(0) & =-\frac{1}{4 \pi} \int \hat{V}(k) \hat{K}_{\mathrm{eff}}(k) k^{2} d k \\
& =-\frac{1}{4 \pi} \int \hat{V}(k) J_{0}(k a) k^{2} d k .
\end{aligned}
$$

In the linear approximation the width of a stationary Gaussian wave function $u(x)=u_{\infty} \exp \left(-x^{2} / x_{\infty}^{2}\right)$ in the quadratic trap (17) is
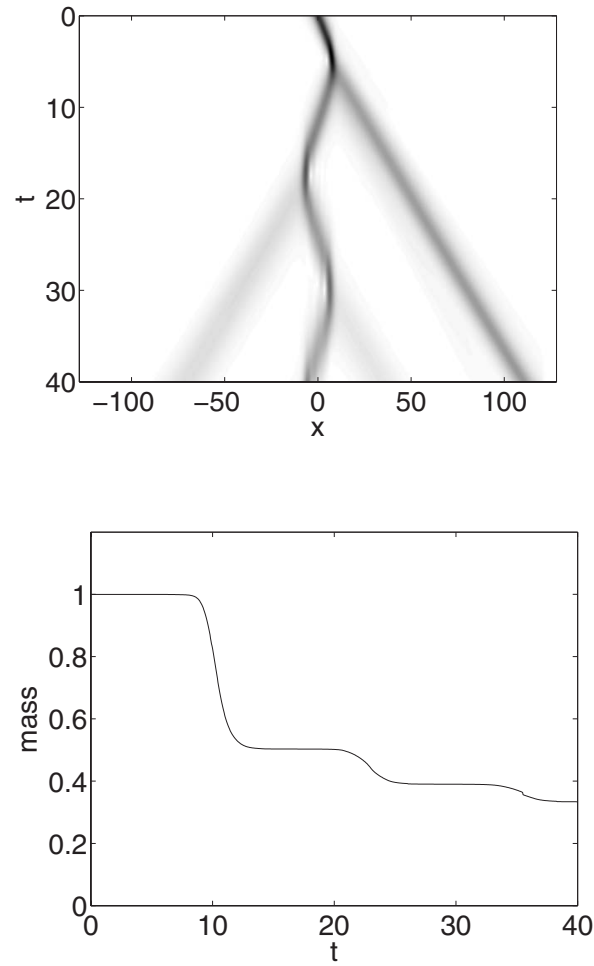

FIG. 6. Soliton profile in the stationary trap $V[x-10 \sin (50 t)]$ (top). Mass decay (bottom). Here the initial velocity parameter is $\mu_{0}=0.5$.

$$
x_{\infty}=\sqrt{2} V_{1}^{-1 / 4}
$$

This result is in good agreement with the numerically observed asymptotic wave function in Fig. 8, where $x_{\infty} \simeq 5$. It is possible to take into account both the cubic nonlinearity and the quadratic trap by using the variational approach with a chirped Gaussian ansatz [21],

$$
u(x, t)=A(t) \exp \left[-\frac{x^{2}}{w(t)^{2}}+i b(t) x^{2}\right]
$$

Substituting this ansatz into the average Lagrangian of Eq. (7) with Eq. (17) and equating to zero its variations, we arrive at the following variational equation for the width $w$ :

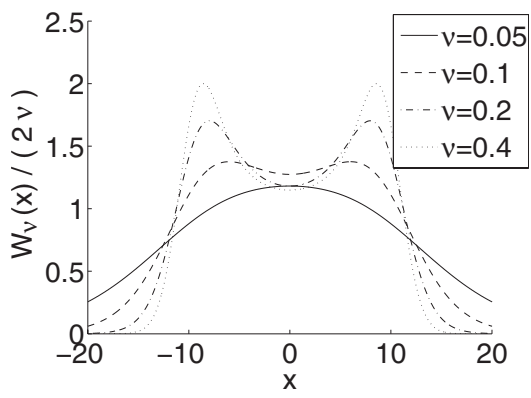

FIG. 7. Potentials $W_{\nu}(x)$ for the quasiparticle motion (12) for different values of $\nu$. Here $V(x)=10 \exp \left(-x^{2} / 4\right)$ and $f(\tau)$ $=10 \sin (2 \pi \tau)$. The local minimum vanishes when $\nu<0.07$. 

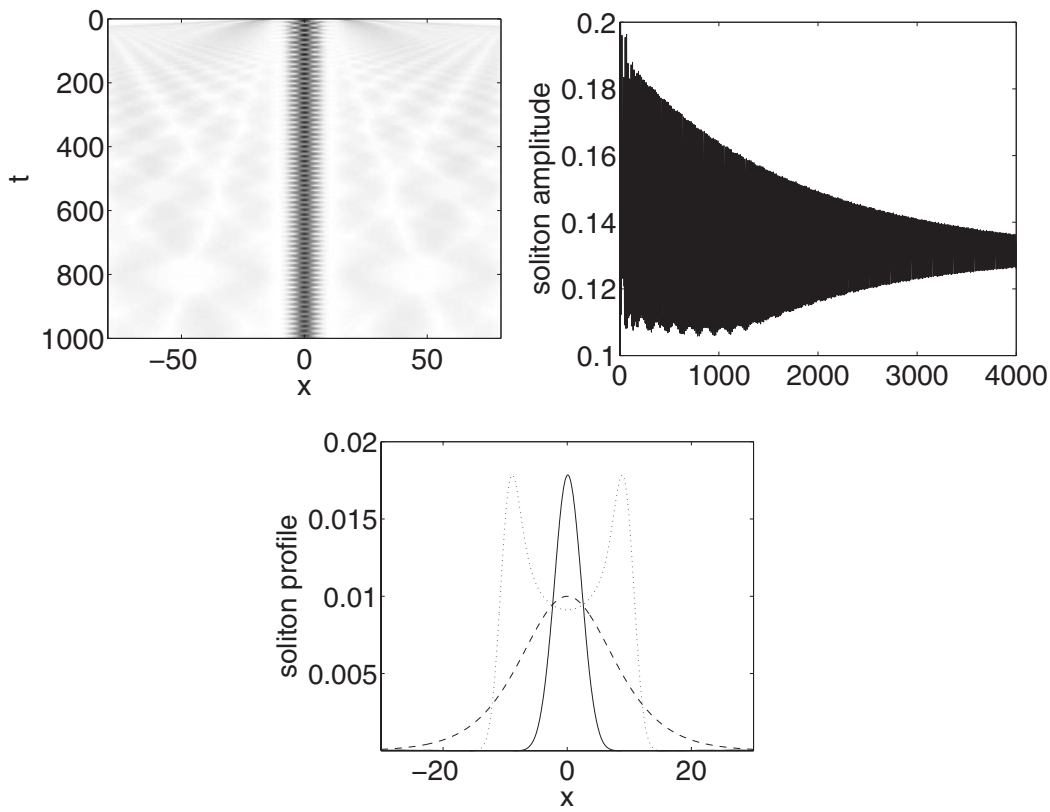

$$
w_{t t}=\frac{16}{w^{3}}-\frac{8 \mathcal{E}}{\sqrt{\pi} w^{2}}-4 V_{1} w,
$$

where the conserved quantity is the number of atoms $\mathcal{E}=\int|u|^{2} d x=\sqrt{\pi / 2}|A|^{2}(t) w(t)$. This second-order ordinary differential equation describes the periodic oscillations of the wave function observed in the numerical simulations of Fig. 8. It can be used also to predict the width of the stationary Gaussian wave function $u(x)=u_{\infty} \exp \left(-x^{2} / x_{\infty}^{2}\right)$ in the nonlinear regime,

$$
x_{\infty}=2^{5 / 4}\left(u_{\infty}^{2}+\sqrt{u_{\infty}^{4}+8 V_{1}}\right)^{-1 / 2},
$$

which is very close to the value (18) obtained in the linear approximation.

\section{ASYMMETRIC TRAPS AND QUANTUM TUNNELING}

It is possible to generate asymmetric potentials by the management technique discussed in the paper. For instance, if the barrier position $f(\tau)$ is of the form

$$
f(\tau)=a-\frac{a}{2}[1+\sin (2 \pi \tau)]^{2}
$$

then the convolution kernel in Eq. (8) is given by

$$
K_{\mathrm{eff}}(x)=\frac{1}{2 \pi} \frac{1}{(a-x)^{3 / 4}(\sqrt{2 a}-\sqrt{a-x})^{1 / 2}} \mathbf{1}_{(-a, a)}(x) .
$$

The corresponding effective potential $V_{\text {eff }}$ and quasiparticle potential $W_{\nu}(x)$ for a Gaussian barrier potential $V$ are plotted in Fig. 9, which shows the existence of an asymmetric trap.

The dynamics of a soliton in such a trap is very similar to the one in a symmetric trap. In particular, if the initial soliton is centered at 0 with zero velocity, then we do not observe any soliton tunneling. As shown in Fig. 10 the soliton emits radiation that escapes the effective trap $V_{\text {eff }}$ and transforms into a breather whose center corresponds to the minimum of
FIG. 8. Top left: Soliton profile in the case $\nu_{0}=0.05, \quad \mu_{0}=0, \quad V(x)=10 \exp \left(-x^{2} / 4\right), \quad$ and $f(\tau)=10 \sin (2 \pi \tau)$. Top right: Soliton amplitude vs time. Bottom: the input profile $|u(x, 0)|^{2}$ is plotted in dashed lines, the output profile $|u(x, t)|^{2}$ is plotted in solid lines at time $t=4000$, and the effective potential $V_{\text {eff }}$ (up to a multiplicative constant) is also represented in dotted lines. the effective trap $V_{\text {eff. The breather slowly converges to a }}$ stationary Gaussian-type wave function.

It is relevant to compare these results with recent works on soliton tunneling with sub-barrier energies $[13,14]$. The motion of topological soliton in an asymmetric potential well with a finite barrier has been investigated. When the width of the well is larger than the soliton width and the width or height of the barrier is large, the soliton dynamics is the one of a classical particle. When the soliton width is comparable
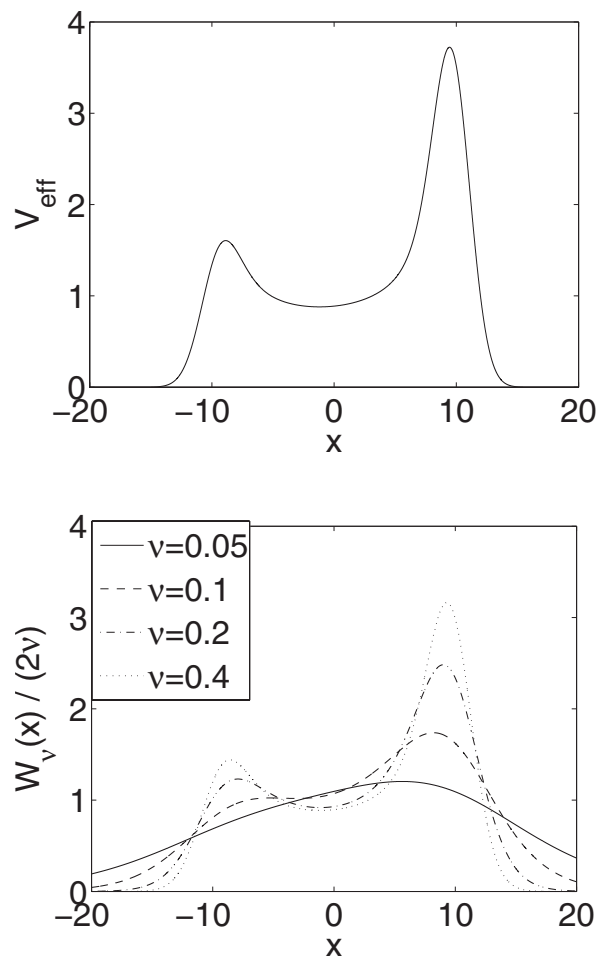

FIG. 9. Effective potentials $V_{\text {eff }}(x)$ for the averaged equation (7) and $W_{\nu}(x)$ for the quasiparticle motion (12). Here $V(x)=10 \exp \left(-x^{2} / 4\right)$ and $f(\tau)=10-5[1+\sin (2 \pi \tau)]^{2}$. 

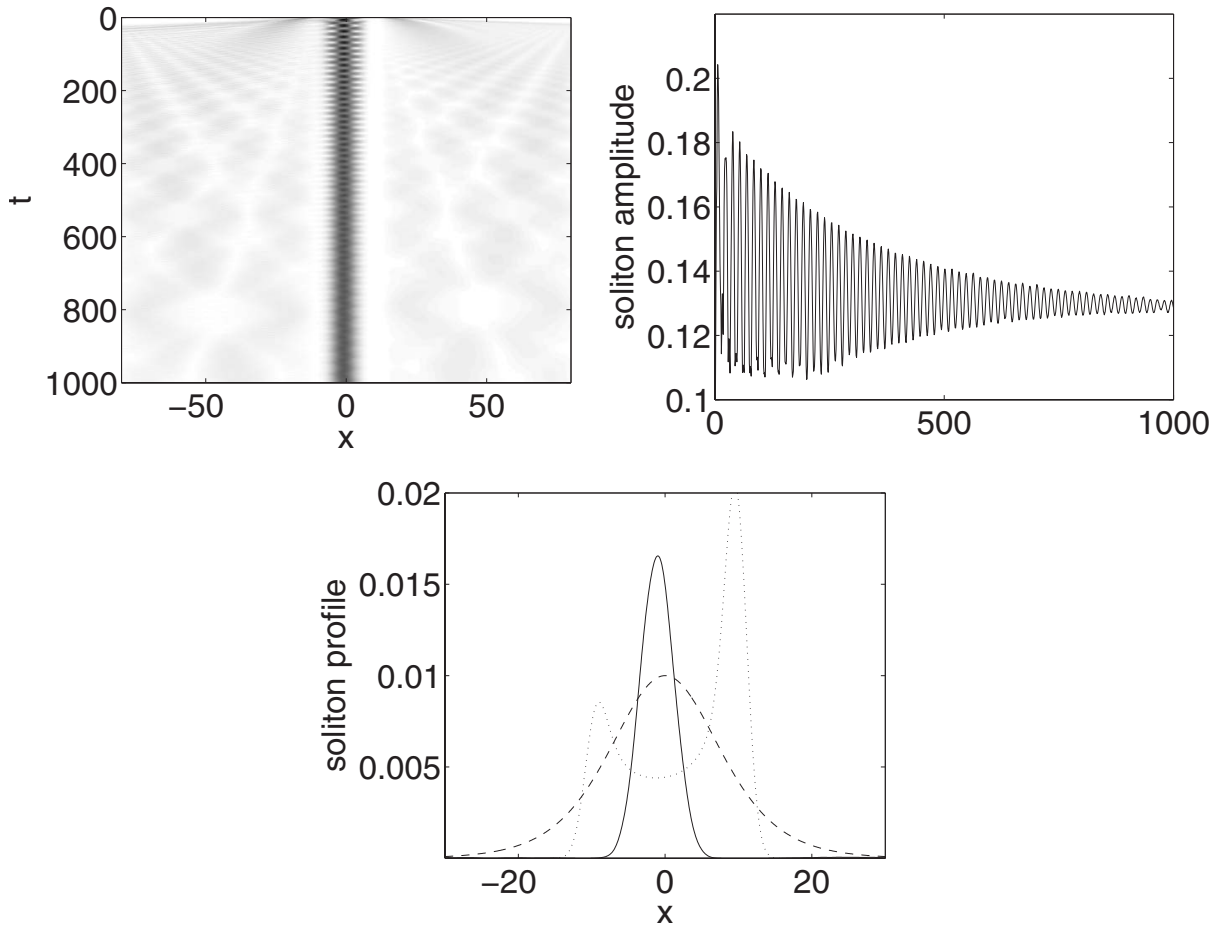

FIG. 10. Top left: Soliton profile in the case $\nu_{0}=0.05, \mu_{0}=0$, $V(x)=10 \exp \left(-x^{2} / 4\right)$, and $f(\tau)$ $=10-5[1+\sin (2 \pi \tau)]^{2}$. Top right: Soliton amplitude vs time. Bottom: input profile $|u(x, 0)|^{2}$ (dashed lines), output profile $|u(x, t)|^{2}$ (solid lines) at time $t$ $=1000$, and effective potential $V_{\text {eff }}$ (dotted lines). with the well width, soliton tunneling with sub-barrier kinetic energies can be observed. Our observations show that, if the NLS soliton is narrow, then soliton tunneling is absent, which is analogous to the topological soliton dynamics case [13]. Increasing the soliton width we observe the continuous shedding of a small amount of radiation from the soliton, which leads to the formation of a breather-type solution that exists for long times in the dynamically generated potential.

\section{CONCLUSION}

In conclusion we have studied the propagation of a bright matter-wave soliton through a barrier potential with a rapidly oscillating position. We have shown that the soliton can be dynamically trapped by such a potential if its width is smaller than the trap width and if its initial velocity is below a critical value predicted by the perturbed IST theory. If the soliton width is comparable to the trap width and its initial velocity is zero, then tunneling radiation emission leads to the generation of a breather in the effective trap.

It would be interesting to extend this model to the case of a standing well and an oscillating barrier with a large oscillation amplitude. In this case the effective potential has the form of a well between two opaque barriers. Applying the results obtained in Ref. [15] we can expect for the transmission of the nonlinear matter waves such phenomena as the instability and quantum turbulence on the time scale of the quantum dwell time in the well. Another physically relevant system is an oscillating barrier enclosed in a broad well. The effective potential in this case simulates a three-well, twobarrier heterostructure and effective nonlinearities can exhibit chaotic dynamics in the tunneling process [16]. These problems require a separate investigation.

\section{ACKNOWLEDGMENT}

F.Kh.A. is grateful to FAPESP for the partial support of his work.
[1] R. Landauer and Th. Martin, Rev. Mod. Phys. 66, 217 (1994).

[2] D. H. Dunlap and V. M. Kenkre, Phys. Rev. B 34, 3625 (1986).

[3] W. K. Hensinger et al., Nature (London) 412, 52 (1993).

[4] D. A. Steck, W. H. Oskay, and H. G. Raizen, Science 293, 274 (2001).

[5] V. Averbukh, S. Osovski, and N. Moiseyev, Phys. Rev. Lett. 89, 253201 (2002).

[6] F. Kh. Abdullaev and R. Galimzyanov, J. Phys. B 36, 1099 (2003).

[7] M. L. Chiofalo, M. Artoni, and G. C. La Rocca, New J. Phys. 5, 78.1 (2003).
[8] D. Embriaco, M. L. Chiofalo, M. Artoni, and G. L. La Rocca, J. Opt. B: Quantum Semiclassical Opt. 7, S59 (2005).

[9] R. Dum, A. Sanpera, K. A. Suominen, M. Brewczyk, M. Kus, K. Rzazewski, and M. Lewenstein, Phys. Rev. Lett. 80, 3899 (1998).

[10] S. Longhi et al., Phys. Rev. Lett. 96, 243901 (2006).

[11] Y. B. Gaididei, P. L. Christiansen, P. G. Kevrekidis, H. Buttner, and A. R. Bishop, New J. Phys. 7, 52 (2005).

[12] K. Bongs et al., Phys. Rev. Lett. 83, 3577 (1999).

[13] J. A. Gonzalez, A. Bellorin, and L. E. Guerrero, Phys. Rev. E 60, R37 (1999).

[14] G. Kalbermann, Phys. Rev. E 55, R6360 (1997). 
[15] M. Ya. Azbel, Phys. Rev. B 59, 8049 (1999).

[16] G. Jona-Lasinio, C. Presilla, and F. Capasso, Phys. Rev. Lett. 68, 2269 (1992).

[17] V. I. Karpman, Phys. Scr. 20, 462 (1979).

[18] Yu. S. Kivshar and B. A. Malomed, Rev. Mod. Phys. 61, 763 (1989).
[19] F. Kh. Abdullaev, S. A. Darmanyan, and P. K. Khabibullaev, Optical Solitons (Springer, Heidelberg, 1993).

[20] G. Theocharis, P. Schmelcher, P. G. Kevrekidis, and D. J. Frantzeskakis, Phys. Rev. A 72, 033614 (2005).

[21] D. Anderson, Phys. Rev. A 27, 3135 (1983). 